\title{
Est-il possible de dire l'éthique de la proximité? Contribution au dossier Kierkegaard - Levinas
}

\author{
Dominic DesRoches
}

On sait que Levinas a été influencé par la pensée de Kierkegaard ${ }^{1}$. On le sait parce que Levinas, en plus de se rapporter à Kierkegaard dans Totalité et infini, lui a consacré deux textes dans Noms propres. On sait également que les deux auteurs, partant de méthodes différentes (la phénoménologie n'est pas la pensée de l'existence), partagent des thèmes philosophiques communs : l'individu, la distance, l'infini et le témoignage, par exemple. Ce que l'on sait moins, par contre, c'est qu'une lecture attentive des écrits dans lesquels Levinas pense avec Kierkegaard peut nous en apprendre beaucoup sur les limites du langage et le problème de la distance pour l'éthique, c'est-à-dire l'éthique de la proximité.

Dans cet article, nous proposons une discussion sur la manière dont l'éthique requiert une attention particulière au langage, au Dit et au Dire, à ce qui est dit et à ce qu'il est impossible de dire sans réduire le Dire au Dit, pour reprendre ici les termes de Levinas. Ainsi voulons-nous mieux saisir les liens entre le langage et l'éthique, qui est un problème de distance, afin de montrer que Levinas, souvent très proche d'un Kierkegaard qu'il critique, s'accorde pourtant avec lui pour réfléchir sur les conditions d'une éthique de la proximité. Dans un premier temps, nous situons la pensée éthique de Levinas en exposant sa critique de l'ontologie, qui constitue l'objectif central de sa philosophie. Dans un second temps, nous précisons les points de contact entre les deux pensées au sujet de l'éthique. Dans un troisième temps enfin, nous tirons les 
leçons qui s'imposent au sortir d'une telle confrontation. Celle-ci nous aura permis de voir les différences entre les deux éthiques, de voir que l'éthique appelle un nouveau langage qui refuse la possession et comment les auteurs envisagent et développent une éthique de la proximité. Le dialogue reposera surtout sur les deux livres les plus marquants de Levinas : Totalité et infini (1961) et Autrement qu'être ou au-delà de l'essence (1974). Il va sans dire que nous accordons une valeur de référence aux quelques pages des Noms propres où Levinas se positionne par rapport à Kierkegaard à propos de l'existence et de l'éthique ${ }^{2}$.

\section{La critique de l'ontologie}

La philosophie de Levinas se présente comme une critique de la pensée occidentale comprise comme métaphysique, comme totalité. Levinas a pour projet de dénoncer toute philosophie qui réduit la vérité à quelque chose qu'il faut conquérir, dominer et posséder. L'ontologie s'inscrit dans le langage à partir du schème de la réponse à une question, la réponse apparaissant comme essence ou concept. Or le concept s'entend toujours dans un système. D'après Levinas, l'attitude philosophique conduit à limiter l'expression de l'altérité et ramène indistinctement tout au même, au régime de l'ontologie, c'est-à-dire que l'altérité tend à disparaître dans l'hégémonie du même, de l'être envisagé de manière verticale, de l'être vu comme vainqueur du combat métaphysique contre l'autre.

Levinas n'accepte donc pas que le langage associe la vérité au même, laissant par là l'Autre

dans l'oubli. À la conscience de soi et au savoir propre de soi, il oppose ainsi l'extériorité, celle qui ne se laisse pas réduire à l'intériorité pure du moi. C'est dire que la vérité véhiculée par l'extériorité demeure irréductible à la pensée (et au langage) qui la conçoit. Levinas en vient à reconnaître dans l'autre personne l'«Autre » par excellence, un Autre qui doit refuser de se 
laisser conquérir, un Autre qui mérite son titre d'altérité. Or cette résistance de l'Autre au Même, explique Levinas, prend sa véritable signification lorsqu'elle se voit comprise comme une résistance à une forme de violence (Totalité et infini 173). Cette thèse forte s'inscrit dans le cadre d'une éthique qui implique la séparation et la responsabilité prise à l'égard de la différence. Elle se comprend dès lors comme une «philosophie première », c'est-à-dire sous la forme d'une pensée éthique qui refuse d'être subordonnée à l'ontologie (281). Il s'agit donc de reconnaître l'antériorité de l'éthique ainsi que la prééminence de l'Autre sur Moi et, par conséquent, de l'altérité sur le mouvement langagier courant de l'essence, donc de l'être.

Ce faisant, une telle éthique requiert un nouveau langage capable de se distinguer de l'ontologie. Cette nouvelle pensée, sensible à la puissance de la rhétorique, jouera de l'hyperbole et aura pour objectif de montrer la dérivation du discours ontologique à partir du discours de l'éthique. À cet effet, Levinas devra recourir aux multiples possibilités du témoignage de la vérité. Or pareille éthique appelle ainsi une question précise, comme le remarque le commentateur danois P. Kemp : «Comment ce témoignage de l'éthique peut-il s’exprimer dans notre langage, un langage qui vise à posséder et dominer les choses? » («Éthique et langage » 189). Cette réponse, comme le suggère Kemp, est à chercher dans l'autre grand ouvrage de Levinas, Autrement qu'être ou au-delà de l'essence, paru treize années après Totalité et infini. Ce changement de cadre nous permettra d'entrer pas à pas dans la problématique de la distance que pose le langage chez Levinas.

Dans Autrement qu'être, le problème central semble être celui de la substitution. En effet, si la subjectivité n'est pas reconnue comme la conscience de soi qui se pose elle-même, Levinas fait voir qu'elle est un rapport à l'Autre dans la proximité, selon lequel l'Autre est toujours proche de moi de telle manière qu'il me rend responsable de lui. C'est ce qui amène Levinas, 
allergique à la domination, à dire que l'individu que je suis est littéralement un « otage » de l'Autre. Pareille expression a toutefois de quoi laisser perplexe, surtout dans le cadre d'une philosophie qui vise à nous libérer des abus de pouvoir du discours ontologique. Sans surprise, Ricoeur y verra un danger, à savoir celui de l'extrême passivité, condition sous laquelle l'éthique paraît négative et incapable de reconnaître le sujet à la racine de l'action. Il s'agit de passer du «pour soi » de la conscience à un «pour l'autre» (un «tout pour tous » pour parler avec Dostoïevski dans Les frères Karamazov, que Levinas aime à citer) que le visage doit révéler. Or pour Levinas, personne ne peut se substituer à moi (Autrement qu'être; voir Ricoeur, Autrement. Lecture d'Autrement qu'être). Dès lors est-il conduit à défendre, contre la métaphysique classique, l'idée (pas neuve, nous le savons en lisant Kierkegaard) que je suis unique, l'irréductible et le non-interchangeable par excellence, tandis que je dois me «substituer » à tous.

Mais Levinas n'en reste pas là et poursuit dans la terminologie de la violence. Pour lui, l'individu unique que je suis est en vérité un persécuté, c'est-à-dire « celui qui est expulsé de son lieu et qui n'a que soi à soi »(Autrement qu'être 155). Cette phrase cache-t-elle plus qu'une métaphore? L'homme est le non-lieu, poursuit Levinas, le non-concept par excellence. L'humanisme visé ici est d'évidence un humanisme marqué par la Seconde Guerre mondiale. Néanmoins, enregistrons la question suivante : comment le langage parvient-il à exprimer ce moi? Comment le langage, qui vise l'universalité, arrive-t-il à rejoindre un moi qui se manifeste comme «otage» de l'Autre? Ou comme irremplaçable et irréductible à toute ontologie? Analysons le langage pour tenter de répondre à cette question.

Le langage, indique Levinas, est ce qui exprime l'essence des choses, ce qui persiste et résiste. Il est une thématisation de la réalité. Cette thématisation, comme savoir, par exemple, 
résulte d'une prise de distance qui vise à détruire l'étrangeté des choses pour nous. Or si l'ontologie s'intéresse à l'être (inter-esse), à ce qu'on pourrait appeler la victoire de l'être sur l'autre, elle n'est pas en mesure de dire la vérité éthique, car celle-ci repose sur un passage à l'autre que Levinas nomme, après le Platon du dixième livre de la République, l'«autrement qu'être ».

Le constat est donc sans appel : l'éthique requiert un nouveau langage parce que sa vérité ne se laisse pas réduire à la domination du concept. Toujours selon Levinas, cet Autre du langage ne peut être autre que la parole vivante possible dans le langage — le Dire, qui ne se subordonne jamais au Dit. L'idée ici, c'est de déjouer toute capture du Dire par le Dit afin d'éviter que le Dire ne devienne une annexe du Dit. Car l'être est ce qui est d'ores et déjà thématisé, déjà Dit, donc conceptualisé. Ce Dit est manifestement en retard et à distance du Dire, le langage que recherche une éthique non métaphysique. L'auteur d'Autrement qu'être insiste : autrui ne peut pas être dit parce qu'il se situe «au-delà de cette essence », l'idée de l'otage inversant d'ellemême ce rapport. Voilà pourquoi la subjectivité éthique s'exprime dans un Dire qui, par rapport à l'être, fait «ex-ception », une exception qui doit être entendue comme le lieu de la proximité (Autrement qu'être 18). Ainsi exprimée, la pensée éthique de Levinas retrouve l'idée de distance que nous savons indétachable de toute éthique philosophique.

Cela dit, reprenons notre question un peu comme l'éthique doit être reprise à nouveau. Notre question devient: comment exprimer l'éthique si le langage butte sur des mots déjà utilisés, si l'altérité est maintenue à distance par cet usage? User de mots déjà utilisés, n'est-ce pas s'exposer à communiquer avec des mots déjà morts? Si le sens des mots ne traduit plus l'altérité, tout est raté. Car si le mot identifie, l'éthique recherchant l'autre du concept devient 
alors impossible. Levinas ne semble pas en mesure de répondre à cette question sans tomber dans certaines « contorsions langagières », c'est-à-dire sans avoir à « dédire » :

L'autrement qu'être s'énonce dans un dire qui doit aussi se dédire pour arracher ainsi l'autrement qu'être au dit où l'autrement qu'être se met déjà à ne signifier qu'un être autrement. L'au-delà de l'être que la philosophie énonce — et qu'elle énonce en raison de la transcendance même de l'au-delà — tombe-t-il sans pouvoir s'en dépêtrer dans les formes de l'énoncé ancillaire? (Autrement qu'être 19-20)

Ce que nous enseigne ce passage délirant, c'est que le « dédire » de l'éthique correspond, pour reprendre l'expression de Ricoeur, à « une trahison de la trahison », la trahison du Dire dans le Dit, que souligne la grande difficulté pour cette éthique de se thématiser sans tomber dans un autre Dit! (Autrement 9). Par ce détour dans les contorsions langagières visant à arracher le Dire aux rets du Dit, Ricoeur voit l'enjeu du livre. Pour notre part, nous sommes d'avis que cette remarque de Ricoeur illustre bien tout l'embarras de penser hors de la métaphysique sans emprunter aucun concept métaphysique. Selon Levinas, le sujet résiste à la condition de se concevoir comme Dire, car derrière l'énoncé de l'être, le Dire déborde l'être qu'il thématise pour énoncer autrui. Ainsi, l'éthique lévinassienne est conduite devant un paradoxe, devant un hic, ou du moins un problème de cercle : peu importe ce qu'elle cherche à dire dans la primauté du Dire, ne fait-elle pas ce qu'elle dénonce en se présentant par le Dit, par la médiation de l'écriture? L'éthique de Levinas ne thématise-t-elle pas déjà le Dire? Comment sortir de cet évident problème méthodologique?

Face à ce cercle embarrassant, l'auteur d'Autrement qu'être soutient que l'on peut retrouver le Dire grâce aux «traces» qu'il a «imprimées » dans le Dit. Ce qui signifie qu'il n'est pas paradoxal selon lui d'effectuer une phénoménologie du Dire à partir du dit. La remontée vers le Dire s'effectue au moyen d'une réduction phénoménologique où l'indescriptible se décrit (Autrement qu'être 60-67). Ainsi, la signification du Dire va au-delà du 
Dit parce que le dit - le mot - est plus qu'un signe, à la fois il proclame et consacre une identification de ceci à cela dans le déjà dit. On voit que la phénoménologie veut accéder à ce «quelque chose » fixé au présent pour l'arracher à la labilité du temps. La notion de trace a pour fonction de donner à penser une temporalité soustraite à l'emprise du présent. Il s'agira d'atteindre le Dire avant le Dit pour y soustraire ce Dit lui-même. Si l'on maintient l'antériorité de l'éthique sur l'ontologie, c'est parce qu'il faudra trouver la signification du Dire (de la responsabilité) avant qu'il soit thématisé. C'est donc dans une diachronie accompagnant la réduction que se trouve la possibilité de dire l'autrement qu'être (75-83). Cela précisé, passons sans plus tarder aux rapports avec l'éthique de Kierkegaard. Nous nous bornerons ici à quelques points de comparaison au centre desquels le langage joue un rôle décisif afin de mettre l'accent sur la distance et la proximité.

\section{Les rapports à l'éthique de Kierkegaard}

\section{(a) Subjectivité et système}

Dans Totalité et infini, Levinas nous met en garde contre une filiation erronée entre sa pensée et celle de Kierkegaard concernant la subjectivité. Contrairement à celle-ci, il insiste (en termes souvent proches de Kierkegaard, cependant) sur l'extériorité :

L'absolument Autre - dont la philosophie de l'immanence surmonte sur le plan prétendu commun de l'histoire - conserve sa transcendance au sein de l'histoire. Le Même est essentiellement identification dans le divers, ou histoire, ou système. Ce n'est pas moi qui se refuse au système, comme le pensait Kierkegaard, c'est l'Autre. (10)

En épigone de J. Wahl (Études kierkegaardiennes) ${ }^{3}$, Levinas reproche à Kierkegaard de tout ramener au sujet dans l'opposition au Système et de faire de ce sujet une « existence tendue sur elle-même, ouverte sur l'extériorité dans une attitude d'impatience et d'attente, impatience 
que l'extériorité ne peut pas satisfaire », c'est-à-dire, écrira-t-il dans Noms propres, qu'« elle [la subjectivité] est une identification du même dans son souci pour le même. Elle est égoïsme » (Totalité et infini 79; Noms propres 101). L'égoïsme est un processus de dépendance qui transforme l'altérité en même. Or Levinas ne règle-t-il pas le cas de Kierkegaard un peu rapidement, ce dernier ayant surmonté l'égoïsme dans les Euvres de l'amour? N'est-il pas luimême impatient? Ne distinguant pas Kierkegaard de ses pseudonymes (surtout le Johannes de Silentio de Crainte et tremblement du Johannes Climacus des Miettes), Levinas ne va-t-il pas un peu trop vite? Ne fait-il pas violence à Kierkegaard et à la complexité de ses pseudonymes en affirmant, sans avoir consulté les Samlede Varker et les Papirer, que la pensée de la subjectivité kierkegaardienne est violente ${ }^{4}$ ? N'est-ce pas la pensée non métaphysique lévinassienne qui dépend de l'énonciation existentielle et l'associe trop rapidement, en partie grâce à la lecture de Wahl, à une pensée métaphysique que Kierkegaard croit avoir déliée avec la répétition (Gjentagelsen)? Voir dans la subjectivité éthique proposée par les auteurs pseudonymes kierkegaardiens une tension sur soi, qui existe, reconnaissons-le, ne conduit pas à faire du sujet existentiel un banal égoïsme ${ }^{5}$. Toutefois, la fin de Totalité et infini nous indiquera que Levinas ne réussit pas à voir Kierkegaard en dehors de son opposition au système de Hegel. N'est-ce pas alors que la réponse à la question ontologique s'énonce en essence, «quiddité », ou en concept, lequel ne peut exister autrement que dans un système de pensée? Ces questions devant être posées, tentons à présent de mieux comprendre le dialogue entre Kierkegaard et Levinas en donnant la parole au premier. 


\section{(b) Silence, thématisation et témoignage}

On se souviendra que l'éthique est comprise comme générale (comme ce qui se produit dans l'ensemble des cas) dans les textes pseudonymes de 1843-1844, et qu'elle ne peut garder sa validité lorsque Dieu exige le sacrifice du fils. Tel est bien le paradoxe devant lequel est placé Abraham, dans Crainte et tremblement, et qu'il est incapable de dire. Ici, c'est non seulement la pertinence, mais la valeur elle-même du silence pour la pensée et l'intériorité qui est soulignée. Cependant, lorsque Levinas décrit la subjectivité comme unique, comme irremplaçable et irréductible à toute thématisation afin d'éclairer la force du Dire, ne le fait-il pas dans le sillage de Johannes de Silentio, l'auteur de Crainte et tremblement? En effet, lorsque Levinas doute de la possibilité du Dire, du langage vivant, face à la réalité, il emprunte non seulement le vocabulaire du pseudonyme de Kierkegaard, mais il reprend au siècle suivant le même problème : comment mettre au langage l'exception, le singulier, c'est-à-dire ce qui échappe par définition au langage? Car si, chez Levinas, le Dire est irréductible au Dit, cela signifie que tout langage, qui procède par généralisation, ne peut que ruiner sa propre valeur. Le général (ou le langage) ne peut jamais rendre compte du particulier, comme l'avait relevé Kierkegaard contre Hegel. Kierkegaard, et Levinas le sait sans doute, opposait la parole vivante (livende Ord) et le silence à toutes les philosophies de la totalisation.

Or Kierkegaard avait bien vu le problème de mettre au langage ce que le langage ne peut pas par lui-même supporter. Comme l'a relevé M. Weston dans son article sur Levinas et Kierkegaard, la thématisation manque ce qu'elle thématise elle-même ${ }^{6}$. La formulation du Dire dans le Dit manque sa visée philosophique, «Philosophy always comes too late » (elle arrive toujours trop tard), pour reprendre le titre de l'article de Weston ${ }^{7}$. Dans le cas d'Abraham, la vérité qu'il aurait à dire déborde le dicible — ne pouvant parler, contrairement au héros tragique, 
sa responsabilité se trouve dans le fait qu'il respecte la Parole, devant Dieu (for Gud), bien qu'il soit confiné au silence absolu. Contre le langage totalisant (notamment de Hegel et ses disciples danois), Kierkegaard opposait le silence dès 1843, parce que la spéculation cherchait, sans se rendre compte de ses limites, à thématiser le paradoxe. Elle prétendait dire ce que le langage, lui, refusait. La pensée idéaliste commettait une grave erreur : elle voulait dire ce qui échappe au dit. Pour l'auteur Johannes de Silentio, le silence exprimait la limite du dire et donnait le ton fondamental à partir duquel toute parole pouvait être entendue.

Cette manière kierkegaardienne de poser le problème nous conduit à la question du témoignage, car pour Levinas, le témoignage est la modalité par laquelle l'Infini, qui échappe au langage, passe dans le fini. L'idée de l'infini en nous dépasse toujours son idée : elle «permet de penser plus que ce qu'elle pense ». Il y a ici expérience de l'Autre sans qu'il puisse être intégré au même. Le témoignage, dans ce contexte, doit rendre accessible l'idée d'infini en tenant l'extériorité de l'Autre à distance du même. Mais à l'inverse de Kierkegaard cependant, Levinas conçoit le silence comme l'«envers de la parole », c'est-à-dire comme un vide qui, provenant de l'intériorité, menace cette dernière ${ }^{8}$. Pour Kierkegaard, l'actualité du témoignage se pose autrement. Si la communication éthico-religieuse doit s'établir au plan de l'existence, dans le médium de l'être, elle doit alors s'achever dans l'acte même de témoigner. Le témoignage apparaît comme « une forme de communication qui se retrouve exactement au milieu, entre la communication indirecte et directe»(Papirer X I A 235 [1849]). Ayant la forme d'un engagement par rapport à la foi, il ne peut apparaître qu'indirectement. Le témoignage est indirect dans un sens plus chrétien encore, puisque ni le secret de l'intériorité, qui est un rapport unique à Dieu, ni le mystère de Dieu ne sauraient se communiquer directement. Voilà en quel 
sens, pour Kierkegaard, il est «entre-deux ». Examinons ici les différences entre les pensées de Kierkegaard et de Levinas au sujet de l'éthique et du langage qu'elle requiert.

\section{L'éthique appelle un autre langage}

Que signifie le mot «éthique» chez nos deux auteurs? Dans sa première éthique, Kierkegaard voit l'éthique comme générale (det Almene), c'est-à-dire ce qui arrive le plus souvent. Cette manière de comprendre l'éthique, après la Sittlichkeit hégélienne, certes, ne laisse pas de place pour une «exception» par rapport à la loi morale. Si exception il y a, celle-ci ne peut que s'opposer et ainsi renforcer la loi générale. C'est que le général implique, par opposition, le rapport à un individu, à un cas particulier. Aussi, seule la crise que traverse une figure d'exception peut être interprétée comme une transgression de la règle, et ce, dans le but de renforcer la norme morale elle-même9 .

Or selon Levinas, l'éthique n'est pas de l'ordre du général, quoiqu'elle soit valable pour tous. L'éthique qu'il propose est bien plutôt une tentative pour échapper à toute éthique idéaliste. Contre Kant et Hegel, Levinas trouve dans l'Autre la possibilité de reprise de l'éthique : l'autre, comme «Autre », est l'affaire de toute réflexion éthique, mais une éthique qui refuse le Dit. La remise en question de ma spontanéité naturelle par la présence de l'Autre constitue la délimitation de cette éthique anti-ontologique. Cet « Autre », qui questionne mon être, appelle un nouveau langage. Il appert que ce langage de l'Autre à partir du visage me somme en ma liberté. Partant du visage nu et vulnérable, on pourra dire que ce langage de l'Autre m'interpelle et me commande impérativement avant même de m'obliger à porter secours à autrui. Cette manière neuve d'envisager la pensée éthique, le langage et le rapport éthique dans la proximité repose 
bien entendu sur un dépassement de l'intériorité à partir du visage d'autrui. Levinas écrit justement :

L'éthique signifie pour Kierkegaard le général. La singularité du Moi se perdrait sous la règle valable pour tous. La généralité ne peut ni contenir ni exprimer le secret du moi infiniment indigent et angoissé pour soi.

Le rapport avec Autrui est-il cette entrée et cette disparition dans la généralité? Voilà ce que l'on doit se demander contre Kierkegaard, comme contre Hegel. Si le rapport avec la généralité ne peut pas former une totalité dont les parties se comparent et se généralisent, ce n'est pas parce que le Moi garde son secret dans le système, mais parce que l'extériorité où les hommes nous montrent leur visage fait éclater la totalité. Cet éclatement du système à partir d'Autrui, n'est pas une image apocalyptique, mais l'impossibilité où est la pensée qui réduit tout autre au même de réduire Autrui. Impossibilité qui n'en reste pas à sa signification négative, mais qui aussitôt met en question le Moi. Cette mise en question signifie la responsabilité du Moi pour l'Autre. La subjectivité est dans cette subjectivité seule la subjectivité irréductible qui peut assumer une responsabilité. L'éthique, c'est cela. (Noms propres 107)

Nous retiendrons trois points de cette interprétation de Kierkegaard. (1) En marge du Moi, Levinas voit l'éclatement du système dans le seul visage de l'Autre : la vérité éthique se trouve dans la mise en question opérée par le visage d'autrui. L'éthique, c'est ici la refondation par le visage qui entend (Kemp, L’irremplaçable 94-95; Plourde, Avoir-l'autre-dans-sa-peau 46). Le visage est nu : il n'est pas sujet à la thématisation, car il demeure insaisissable et résiste dès lors à toutes mes tentatives. (2) Cette remise en question de la subjectivité par l'Autre implique, sans plus d'explication, la responsabilité. Contrairement à Kierkegaard qui fonde la responsabilité dans l'effort $d u$ choix de l'autre en moi, Levinas l'établit dans la seule reconnaissance de la différence entre le visage d'Autrui et mon irréductibilité. Et (3) en raison de sa méthode, Levinas ne peut définir la subjectivité que comme « une tension sur elle-même ». De là, il peut conclure que cette tension est égoïsme et qu'elle donne prise à l'irresponsabilitét ${ }^{10}$. La critique qu'il fait de la pensée de Kierkegaard est simple et commode : le soi existentiel kierkegaardien, victime de son secret et de sa passion dans l'intériorité, serait impuissant à 
revenir sur lui-même. Cette subjectivité tendue sur elle-même, incapable de sortir de son égocentrisme, n'arriverait jamais à rompre avec le souci de soi. Si elle ne réussit pas à sortir pour rejoindre autrui dans l'extériorité, elle peut encore moins revenir sur elle-même et accomplir une éthique comme celle de Levinas.

Or, contrairement aux allégations de Levinas, l'œuvre de Kierkegaard montre que cette expression éthique de l'intériorité ne dérape pas dans un idéalisme (qui vise la connaissance) de l'ipséité ni une pensée du Moi sans l'Autre. Au contraire, il appert que l'éthique réside dans cette « tension » entre l'individu et le social, entre l'homme singulier devant aller au bout de lui-même et la communauté à laquelle il appartient. Comme l'a soutenu A. Clair, c'est d'ailleurs ce cadre social qui assure une normativité à l'éthique. Ce qu'oublie Levinas, c'est de rendre à la subjectivité sa valeur hors du système, à partir d'un rapport entre elle et une instance supérieure capable de justifier le rapport à la norme. Cette dérive n'est pas le fruit du hasard, puisque Levinas pense Kierkegaard dans son rapport à Hegel. Pourtant, et l'étude de l'immense corpus est claire, Kierkegaard, bien qu'il mette l'accent sur le choix de la subjectivité, ne pense jamais la subjectivité sans la norme sociale qui la définit. Comme le montre J. Janiaud, «cette éthique générale est au fond une éthique du commensurable, une éthique du sujet s'identifiant à luimême, tenant debout par lui-même aux côtés de son prochain» («"Me voici!" »96). D. Brezis ajoutera non sans raison que «l'écriture biographique » traduit les limites de la critique de Levinas («L'intériorité en question» 17-19). En fait, tous les mécanismes de l'écriture kierkegaardienne (on pense à la stratégie de production, mais aussi à la duplicité de l'œuvre et au travail de la pseudonymie) illustrent que l'œuvre de Kierkegaard est beaucoup plus qu'une réplique au système de Hegel 
S'il est vrai de dire que Kierkegaard et Levinas s'opposent, c'est peut-être dans la mesure où l'éthique est l'enjeu fondamental chez Levinas, alors que Kierkegaard fait appel à un dépassement de l'éthique dans la vérité chrétienne. À ce sujet, cependant, les nuances s'imposent. Car il n'est pas inopportun de rappeler que Kierkegaard distingue, par Haufniensis, une éthique seconde qui, dans le dépassement des lacunes d'une éthique basée sur la métaphysique, se rapproche d'une éthique chrétienne. Mais Kierkegaard dissocie la vérité «éthique », «éthico-religieuse », de la vérité «chrétienne ». Levinas consacre la suprématie à l'éthique qui ne doit plus tomber dans un langage qui réduirait la richesse de l'altérité au même. Les deux penseurs reconnaîtront, avec Wittgenstein notamment, le caractère absolu de l'éthique.

\section{Distance, proximité et pouvoir}

Si Kierkegaard adopte les schèmes de l'ouïe, de l'intériorité cachée et de la communication indirecte, Levinas opte pour la puissance de persuasion du visage d'autrui. Il croit que le visage dépasse l'idée de l'Autre en moi, c'est-à-dire de l'Autre dans le même. Le visage, note Levinas contre l'ontologie, « apporte une notion de vérité qui n'est pas dévoilement d'un Neutre impersonnel, mais une expression » (Totalité et infini 21-22). Le visage demeure l'expression originelle du commandement «Tu ne tueras point! » (Kemp, L'irremplaçable 98). C'est que l'expression du visage supprime toute distance qui voudrait dominer entre l'autre et moi. Elle m'oblige. Elle est un ordre avant la loi des hommes.

Cette expression implique une compréhension du pouvoir, ce qui la rapproche beaucoup de la conception qu'esquisse Kierkegaard dans ses leçons sur La dialectique de la communication. Car ce que le visage présente, c'est une expression qui ne défie pas la faiblesse de mes pouvoirs, mais «mon pouvoir de pouvoir» (Levinas, Totalité et infini 172). Elle modifie 
alors la nature même du pouvoir « qui ne peut plus dès lors prendre mais tuer », car Autrui est un être que je peux tuer, pas seulement une chose que je peux détruire sans conséquence. D'où l'importance du langage de l'Autre : il me rappelle une obligation et me juge, et il m'oblige à lui prêter secours dans la «détresse » (Totalité et infini 170). Chez Kierkegaard, c'était le langage qui jouait le rôle du visage comme don et juge. Levinas a vu dans le visage un langage unique, particulier et irréductible, et telle est ici une grande partie de son originalité en regard de la pensée de l'existence de Kierkegaard.

\section{(a) La dialectique de la distance}

Or c'est encore et toujours sur le thème de la distance que Kierkegaard et Levinas se rapprochent et se distinguent le plus, tout se passant comme si l'enjeu de l'éthique était de donner une compréhension de la distance qui me sépare d'autrui. Dans les Euvres de l'amour, le livre qui accomplit la seconde éthique annoncée dans le Concept d'angoisse, Kierkegaard associait la promesse, qui consiste à tenir parole, à l'exigence éthico-religieuse du «rendre attentif » dans la problématique de la distance. Il écrivait :

Quand un homme me tourne le dos et s'en va, je vois sans peine qu'il s'éloigne; mais s'il s'avise de tourner le visage vers ce dont il s'écarte à reculons et qu'il me salue du geste, du regard et de ses exclamations où il ne cesse de m'assurer qu'il arrive tout de suite et même de répéter sans arrêt «me voici » alors qu'il s'éloigne toujours plus, à reculons, j'ai plus de mal à m'en apercevoir. De même pour l'homme qui, plein de bonnes intentions et prompt aux promesses, s'éloigne continuellement du bien — à reculons. Bonnes intentions et promesses lui font envisager le bien vers lequel il est tourné, alors qu'il s'en écarte de plus en plus, à reculons. (Euvres complètes XIV 86-87 [IX 112-113])

La première chose qui nous frappe, c'est que les thèmes importants de ce passage seront tous repris par Levinas : la distance, le fameux «me voici! » et la promesse, mais dans des sens légèrement différents. Levinas a-t-il médité ce passage, lui qui a connu les œuvres par d'autres? 
Ici, Kierkegaard souligne d'abord toute la difficulté de tenir parole, de répondre à la parole qui oblige, parole d'action qui constitue, dans l'amour, l'accomplissement de la loi. Un « oui » hâtif à une exigence peut nous tenir à distance de cette exigence éthique. Mais il y a plus : dire « oui » à la promesse d'accomplir la loi n'est pas encore action, car la parole, contrairement à l'action, est abstraite - l'enfer est pavé de bonnes intentions. Notre passage montre en outre que le «me voici! », qui est la réponse positive à la promesse, peut aussi nous faire reculer, nous éloigner des autres et nous faire perdre de vue la parole de Dieu. Dire «me voici! » peut être un détour sur le chemin de l'éthique lorsque l'amour n'est pas le seul guide de l'action. Dire «me voici! » et s'éloigner lentement (c'est-à-dire aller dans le mauvais sens) du prochain signifie le contraire de l'éthique (Janiaud, «"Me voici!" » 97-108). Une analyse de ce passage aussi sommaire que la nôtre établit assez clairement que Kierkegaard paraît mettre en garde Levinas par anticipation contre le danger d'omettre les conséquences de la dialectique de la distance éthique ${ }^{11}$. Car approcher la réalité éthique dans son exigence et chercher à construire une éthique de la proximité impliquent qu'une attention particulière soit portée à la question de la distance et à sa dialectique existentielle.

C'est encore sur l'idée de proximité que Kierkegaard et Levinas se touchent et deviennent des complices. Certaines pages d'Autrement qu'être semblent sorties d'un livre de Kierkegaard. Quand Levinas avoue candidement : «nous avons essayé de décrire la subjectivité comme irréductible à la conscience et à la thématisation » ou « qu'en soi, le soi-même est l'un ou l'unique séparé de l'être » (126-135), ce pourrait bien être des lignes extraites du Post-scriptum de Kierkegaard. Nous obtenons un tel résultat entre autres parce que Levinas veut dépasser la pensée de l'existence en appliquant la méthode phénoménologique sur les mêmes thèmes éthiques. Dans Autrement qu'être, il cherche en effet à penser, aux limites de la phénoménologie 
de Husserl, le «sujet de chair et de sang » cher à Kierkegaard ${ }^{12}$. Pour y arriver, il montre que la proximité ne se laisse pas penser avec le vocabulaire de la spatialisation et qu'elle se «donne » plutôt sur le mode de l'inquiétude. La proximité n'est pas espace, mais d'abord souci.

Or c'est lorsque Levinas fait de la proximité la situation de la subjectivité qu'il se retrouve au plus près du Kierkegaard des Euvres de l'amour, l'opus magnum dans lequel il se présente comme le premier auteur à poser le problème de la compréhension à distance (forstaa paa Afstand $)^{13}$ de l'autre. Ayant en tête la proximité du prochain, celui qui échappe à mon ordre et à ma connaissance intéressée, Levinas glisse de l'éthique vers une sphère plus large. L'inquiétude est pour lui ce non lieu, ce hors lieu, ou cette utopie, qui demeure confrontée à l'exigence de la loi dans la proximité :

La proximité n'est pas un état, un repos, mais, précisément, inquiétude, non lieu, hors le lieu du repos, bouleversant le calme de la non-ubiquité de l'être qui se fait repos en un lieu, toujours par conséquent insuffisamment proximité, comme étreinte. «Jamais assez proche », la proximité ne se fige pas en structure sinon, quand représentée dans l'exigence de la justice, réversible, elle retombe en simple relation. La proximité, comme le «de plus en plus proche», se fait sujet. (Autrement qu'être 131)

Dans la proximité se tiennent tout engagement, toute fraternité. Mieux : le sens de l'ouverture et de la relation à autrui est à trouver dans la proximité. Ainsi joue-t-elle un rôle clef chez Levinas : elle est «suppression de la distance» (aussi dans la conscience) qui s'accomplit dans l'impossibilité de m'éloigner d'autrui. Or, dans cette exigeante proximité, mi-étreinte midistance, Levinas craint une obsession violente pour l'Autre, pour le prochain, c'est-à-dire une obsession qui briserait les possibilités éthiques de la proximité elle-même. Si la distance « inquiète » de la proximité construit la subjectivité, lui donne corps, l'obsession par le prochain est non-réciprocité, car elle ne reconnaît pas dans l'Autre, pour reprendre Kemp, un « irremplaçable ». L'obsession est pathologique en ce qu'elle vise à détruire la proximité, et la 
séparation qui la caractérise s'impose comme un mépris de l'éthique. Si la proximité construit la relation, le sujet peut, par son obsession de rapprochement, la briser.

\section{(b) Danger dans la proximité et l'idée d'infini}

Or faisons un pas de plus. Dans sa réflexion, Levinas est si près de la pensée de Kierkegaard qu'il va jusqu'à utiliser ses mots les plus personnels, c'est-à-dire ceux du fameux épisode de «l'écharde de la chair». Si Levinas n'utilise pas la méthode dialectique et qu'il n'a pas une connaissance de première main des Papirer, il n'en cherche pas moins à découvrir l'Autre dans les mêmes sillons que l'auteur danois. Non pas de confession luthérienne protestante, mais juive ${ }^{14}$, Levinas parvient à écrire des lignes «kierkegaardiennes », tout en y ajoutant son grano salis anti-ontologie :

Le discours conscient sait encore dire cette impuissance sans guérir l'affection qui déchire la conscience, sans arracher par l'aveu le grain de folie - écharde dans la chair de la raison — qui est le frémissement de la subjectivité; même chez le philosophe qui en parle et qui, par-delà l'universalité où apparaît l'universalité dite, reste subjectivité obsédée par le prochain. Le Dire où le sujet parlant s'expose à autrui, ne se réduit pas à l'objectivation du même exposé : qu'est-ce qui est donc venu blesser le sujet pour qu'il expose ses pensées ou s'expose dans son Dire?! (Autrement qu'être, 133)

On ne peut que souligner la parenté dans leurs tentatives de penser l'éthique de la proximité à même la blessure de l'existence. Or la question de l'éthique de la proximité peut encore s'interpréter si on la pense avec l'idée d'infini déjà abordée dans Totalité et infini. On comprendra la distance comme préoccupation lévinassienne en analysant cette idée d'infini qu'il reprend à Descartes. Pour lui, l'idée d'infini est l'idée morale de l'absolument Autre, c'est l'ideatum qui dépasse son idée : elle est la séparation du même par rapport à l'autre. On l'a dit plus haut : l'idée d'infini me permet de penser plus qu'elle-même. Ce n'est donc pas un hasard si Levinas écrit dans Autrement qu'être : «La proximité est ainsi une relation avec une singularité 
sans la réduction d'aucun principe, d'aucune idéalité » (127). La proximité seule peut rendre justice à autrui : non seulement le visage expose un sens, mais sa présence m'oblige à une responsabilité qui ne se justifie par aucun engagement préalable. Si le Dire se donne comme le langage de la transcendance, la proximité du visage serait alors l'autre langage qui me permet de découvrir autrui. Pas étonnant, donc, que ce thème soit si déterminant dans une éthique qui recherche un langage respectueux de la différence éthique, d'autant plus que Levinas en fait le titre d'un texte : Langage et proximité. Dans ce texte peu connu de 1967, d'ailleurs, il écrira que ce n'est pas l'individu en tant qu'individu d'un genre, mais plutôt la «singularité unique en son genre» (expression qui n'est pas sans rappeler la pensée de Kierkegaard) qui s'approche d'autrui. Abordons désormais la question de la séparation.

\section{(c) La séparation et le pouvoir}

Ces mots ne sont pas difficiles à interpréter lorsque nous avons en tête que l'infini, pour Levinas, implique le dépassement du subjectif. Quant à la proximité, elle doit être entendue en dehors des catégories métaphysiques: elle est une distance «diminuée » nous rapprochant d'autrui sans le dominer. La question de la distance s'établit sur un réel problème de séparation pour Levinas, car la subjectivation commence dans la séparation. Or les premières lignes de «Existence et éthique », dans Noms propres, étaient déjà limpides au sujet de cette origine :

L'idée forte de l'existence que la pensée européenne doit à Kierkegaard, revient à maintenir la subjectivité humaine - et la dimension d'intériorité qu'elle ouvre - comme absolue, comme séparée, comme se tenant en deçà de l'Être objectif, mais aussi à défendre paradoxalement la position irréductible du sujet contre l'idéalisme qui lui avait cependant préféré un rang philosophique, à partir d'une expérience pré-philosophique. (99) 
Comme le disciple danois de Luther l'avait vu, c'est en se «séparant» des autres que l'homme retrouve sa subjectivité éthique et gagne en intériorité. La séparation soulignée par Levinas ne correspond toutefois pas à celle de Kierkegaard conçue comme «résignation » (Resignation), comme mouvement vers l'intériorité ${ }^{15}$. Au contraire, elle est vie corporelle, puissance et joie. Levinas critique avec raison une certaine absence de corporéité chez Kierkegaard - Kierkegaard ayant lui-même avoué ce défaut plusieurs fois, car la rencontre d'autrui présuppose la sortie hors de soi : l'autre n'est pas physiquement en moi, mais hors de moi. La pensée kierkegaardienne de l'absolu, selon lui, éviterait toute extériorité, comme le note J. Nizet qui découvre Kierkegaard avec Levinas («La temporalité chez Soeren Kierkegaard» $244-245)^{16}$. Ainsi, la séparation chez Levinas veut éviter toute «abstraction » vers le plus petit, qui d'ailleurs est la principale critique d'Adorno à l'endroit de Kierkegaard ${ }^{17}$. S'il y a un mouvement en direction de l'intériorité chez Levinas, il se dirige aussi en direction de la communauté (Totalité et infini 88). En ce sens, Levinas revient à Kierkegaard, car il se voit obligé d'user du processus de la double réflexion ${ }^{18}$. Nous reviendrons plus loin sur ce processus de pensée décisif pour Kierkegaard.

Si l'ontologie doit être débusquée, dit Levinas, c'est d'abord en raison du refus de la distance éthique qu'exige l'Autre. L'ontologie traditionnelle est le discours du pouvoir qui pose une distance neutralisante, celle qui exclut et qui masque la singularité dans l'inquiétude. Autrement dit, l'ontologie visée par la critique radicale de Levinas est celle qui, véloce et impatiente, dominatrice, ne cesse de réduire l'Autre au Même dans une forme de violence. La vérité, qui devrait rapprocher authentiquement dans la distance, est celle du souci et de l'attention; mais cette vérité n'existera cependant pas uniquement dans l'anonymat, la distance à l'égard du nom et de la personne. C'est pourquoi une universalité de l'éthique ne pourra être 
qu'impersonnelle chez Levinas, alors qu'elle implique une tâche plus personnelle chez Kierkegaard. Ce dernier usera de plusieurs pseudonymes pour développer une «première éthique » (dans les textes de jeunesse) et, en même temps, il signera de son nom des discours religieux et approfondira sa «seconde éthique» dans Les auvres de l'amour (des textes autonymes).

\section{Dire de l'éthique, vérité persécutée et transcendance}

Sur la question du Dire de l'éthique, les deux auteurs s'entendent pour voir dans la communication une médiation produite par le langage. Utilisant des signes et des mots, le langage pose une distance qui aliène l'éthique dans la métaphysique et masque la vérité du rapport à l'Autre. Or si, dans la communication directe, les médiations du discours peuvent être complexes, il y aura cependant toujours un lien entre le signifiant et le signifié, c'est-à-dire entre le Dire et le Dit. Kierkegaard, et il est aisé de le comprendre, se serait montré intéressé par une réflexion comme celle qu'exerce Levinas dans Autrement qu'être, car la seule communication éthique possible est indirecte, hors de la symétrie entre le Comment et le Quoi afin de respecter la distance du Dire et du Dit. Kierkegaard, et Levinas aura la même préoccupation, s'intéressait beaucoup à la manière d'approcher et de communiquer la vérité éthico-religieuse. Comme en témoignent ces Leçons sur La dialectique de la communication (1847) culminant dans la distinction décisive entre la communication de savoir et la communication de pouvoir, le Danois réfléchissait aux rapports compliqués entre le «Je », la vérité, le médium et le message. Il était persuadé que son époque avait besoin d'un nouveau « Je » afin que l'on puisse faire entendre une « autre » vérité, une vérité d'action irréductible au savoir et à la communication directe ${ }^{19}$. 


\section{(a) Une nouvelle manière d'approcher la vérité et le paradoxe}

$\mathrm{Au}$ sujet de la vérité, l'article sur Kierkegaard dans Noms propres nous indique clairement l'enjeu. Levinas veut voir dans la position de Kierkegaard un refus de la vérité « triomphante », celle qui s'inspire de la raison pour atteindre l'universalité, au profit de la vérité « persécutée ». La thèse de Levinas est simple : se fondant dans la foi, l'éthique de Kierkegaard se contracte dans une «tension sur soi » qui n'assume pas le passage à l'extériorité et qui culmine dans l'égoïsme. Ainsi subjectivité doit rimer avec souffrance.

Pour comprendre cette critique de Levinas, prenons un sentier peu habituel dans les études entre nos auteurs. Partons de l'Instant afin de montrer la différence qualitative entre le fini et l'infini qui crée le paradoxe et la souffrance. Chez Kierkegaard, l'Instant est paradoxe. Comme l'exposent les ouvrages pseudonymes, l'Instant est la découverte du passage de l'éternité dans le temps. Ce passage, dans les Miettes et le Post-scriptum, est aussi une condition qui produit la conscience de soi devant son «autre ». Ici, l'éternel de la foi est transcendant car il est un rapport à une vérité paradoxale : le croyant doit croire au paradoxe du Dieu-homme. La subjectivité croyante doit s'avancer dans l'incertitude vers son Autre. Or, le sujet entrera en rapport avec Dieu s'il admet au fond de lui la contradiction, c'est-à-dire l'absurde. Dans l'Instant du don, qui est à la fois amour et paradoxe, la foi requiert la volonté de croire à un paradoxe indicible qui heurte de front la raison. Il est, dit un Kierkegaard lecteur de Luther, scandale pour l'intelligence. Ainsi, la vérité se trouve cachée dans le paradoxe et ne se laisse pas cerner par l'entendement. Climacus explique cela dans l'appendice des Miettes :

Si la collision [entre le paradoxe et la raison] se produit sans cette intelligence, la situation qui en résulte est malheureuse [...]. Le scandale peut être considéré comme une preuve indirecte du bien fondé du paradoxe; car le scandale est calcul erroné, il résulte de l'état de non-vérité grâce auquel le paradoxe rebute. [...] Plus l'expression que prend le sandale est profondément ancrée dans la passion, plus aussi se révèle tout ce que le 
scandale doit au paradoxe. Le scandale n'est donc pas la découverte de la raison, loin de là [...] le scandale devient de fait avec le paradoxe; il prend force de réalité : ici nous avons l'Instant autour duquel tout gravite. (CEuvres complètes VII 49-50 [IV 244-245])

Parce qu'elle surgit du scandale et que «tout scandale est un pâtir », la vérité se révélant est souffrante : elle est attente, non reconnaissance, voire un amour malheureux. Kierkegaard est sans aucun doute le premier à parler de la vérité comme non-possession, c'est-à-dire comme un événement qui vient à moi et qui défie mon pouvoir de conquête. Contrairement à la vérité en régime païen, comme chez Socrate qui trouve la vérité au fond de lui par le dialogue, le scandale chrétien repose sur une vérité « tout autre » qui se donne dans une promesse impossible pour la raison. L'homme (chrétien) de Kierkegaard est donc inquiet, souffrant, à l'image de la vérité de l'Instant. Or, comme le dit Levinas, seule la foi est capable d'appréhender une telle vérité. Le croyant ne peut pas se rapporter à une vérité «triomphante», mais seulement à une vérité «persécutée » en accord avec la condition du pécheur, car c'est ici l'incertitude dans la foi qui est l'atmosphère. Relisons à nouveau les propos du Climacus de Kierkegaard :

La foi est l'incertitude objective produite par le choc et le refoulement de l'absurde, incertitude gardée dans la passion de l'intériorité, passion qui est justement le rapport de l'intériorité [Inderlighed] porté à son plus haut degré d'intensité. [...] [L]'incertitude est la marque, et la certitude absolue le signe qu'on ne se rapporte pas à Dieu. (CEuvres complètes XI 288-289 [VII 602-603])

Dans l'incertitude, qui est gardée en l'intériorité (Inderlighed), la foi requiert l'action afin de faire apparaître la certitude. Cette certitude n'est pas science, n'est pas absolue, mais c'est celle du croyant qui croit en vertu de l'absurde (i kraft det Absurde). Parti dans l'incertitude totale, si on se rappelle du Crainte et tremblement de l'autre Johannes, c'est-à-dire en son intériorité confrontée au plus Haut, Abraham retrouva par la foi son fils Isaac.

Ainsi est-il difficile de ne pas voir qu'il y a bien, pour Johannes Climacus, une opposition et une distance entre l'incertitude, qui est la marque de l'absurdité de la foi, et la certitude, qui ne 
peut être absolue. La certitude se trouve dans le saut, l'acte de foi. Ce qui est manifeste dès lors, ce n'est pas tant le triomphe de la certitude, mais bien la lutte infinie contre l'incertitude, ou plutôt dans l'incertitude. Levinas l'avait compris lorsqu'il écrivait dans Noms propres : «dans la croyance, l'existence cherche la reconnaissance [...]. Cette reconnaissance lui est accordée par une vérité elle-même bafouée et non reconnue et toujours à reconnaître » (104). Quand il pense la proximité et la vérité comme non-reconnaissance face à l'Autre, Levinas reprend les préoccupations éthico-religieuses de Kierkegaard, et quand il le critique dans Noms propres, il demeure en filiation avec le pseudonyme des Miettes et du Post-scriptum. Si on comprend mieux la vérité souffrante critiquée par Levinas, il convient d'aborder sa principale critique, celle de l'égoïsme du Moi kierkegaardien.

\section{(b) La tension sur soi et la critique du Moi égoïste}

Dans la foi, la subjectivité a un rapport avec la vérité d'un genre unique, puisque la vérité se dévoile tout en se dissimulant aussitôt. Sa manifestation ne correspond pas à un dévoilement, mais à un voilement. Ce paradoxe, qui rappelle la coïncidence des opposés de Nicolas de Cues utilisée par J. Hamann, rappelle que la vérité n'est pas saisissable par la pensée car elle garde, dans son oscillation, son caractère indicible. Et c'est dans la dissimulation que se trouve la vérité paradoxale. Le sujet est celui-là qui, ne trouvant aucune extériorité satisfaisante qui puisse le contenir, selon Levinas, demeure dans une tension sur soi. Mais si cette tension est comprise comme intérêt infini pour soi, Levinas ne se montre-t-il pas impatient envers Kierkegaard puisque tout inter-esse, bien qu'il soit contraction, se fonde sur un rapport extérieur? L'intérêt est aussi, c'est capital pour toute la pensée de Kierkegaard, la possibilité de la liberté, l'espace pour rencontrer l'autre, c'est-à-dire l'espace propice au retour sur soi dans le redoublement éthique ou 
religieux. La subjectivité reste-t-elle vraiment dans un égoïsme sans fin, comme le croit Levinas? Une lecture attentive nous oblige à la nuance.

Dans la foi dépeinte par Kierkegaard, le sujet n'est jamais assuré de la présence divine. Car dans la foi, la subjectivité se trouve confrontée à un dieu qui se manifeste tout en se voilant et qui renvoie la subjectivité à elle-même, c'est-à-dire à l'incertitude de sa condition comme non vérité. La distance se trouve donc trop grande entre l'intériorité et l'extériorité, d'où la souffrance. La subjectivité séparée, intensité dans l'intériorité selon Climacus, souffrirait de l'impossibilité de sortir d'elle-même.

Mais Levinas poursuit et oublie dans sa critique le travail de la double réflexion qu'accomplit la pensée kierkegaardienne. Il écrit : «La vérité qui souffre n’ouvre pas à l'homme les autres hommes, mais à Dieu dans la solitude », que Levinas nomme, on le sait, « un drame intérieur ». Désormais préoccupé par son mouvement réflexif, Levinas conclut à «l'idée [que] la vérité qui souffre transforme toute recherche de la vérité... en un drame intérieur », et que son « discours [Kierkegaard] tourné vers l'extérieur est colère et invective » (Totalité et infini 82; voir aussi Noms propres 104). Or, que dit la double réflexion? Ce mouvement consiste en un effort de pensée où la réflexion «seconde » apparait contraire à la première (celle qui part de l'intérieur vers l'extérieur) dans la mesure où, rentrant vers l'intériorité, elle vise à l'affirmation de l'acte d'exister. Elle consiste dans le fait de montrer les deux possibilités opposées dans le choix. Si la double réflexion trouve une grande utilité dans une dialectique de la communication, si elle est accueil, c'est parce qu'elle n'oublie jamais l'extériorité (CEuvres complètes X 70 et XVII 122). 


\section{(c) Vérité et transcendance}

À l'aune de la double réflexion, on peut trouver que cette dernière critique de Levinas est décevante. Or dans une conférence de 1968 parue dans son livre Entre nous et intitulée «Un Dieu homme? », Levinas revient saluer la tentative de Kierkegaard pour penser la vérité du prochain sans renoncer à la transcendance. On sent que Levinas est obligé de nuancer sa critique, c'est-à-dire de la préciser. S'il est trop léger de dire que le Moi égoïste de Kierkegaard est responsable du fondamentalisme religieux et des guerres en Europe, il reconnaît cette fois que c'est Kierkegaard qui a le mieux compris la transcendance de Dieu dans le thème biblique de l'humilité (Entre nous 67). Le mode de la vérité comme humilité ne peut plus être associé au pire de l'homme. Revenant sur la distance au sujet du Dieu-homme, il précise sa pensée en ces termes :

Pour que l'altérité dérangeant l'ordre ne se fasse pas aussi participation à l'ordre, pour que demeure ouvert l'horizon de l'au-delà, il faut que l'humilité de la manifestation soit déjà éloignement. [...] La figure conceptuelle que dessine l'ambiguïté — ou l'énigme de cet anachronisme où se fait une entrée postérieure au retrait et qui, par conséquent, n’a jamais été contenue dans mon temps et est ainsi immémoriale, nous l'appelons trace. Mais la trace n'est pas un mot de plus : elle est la proximité de Dieu dans le visage de mon prochain. (Entre nous 68)

Ce passage est riche parce qu'il reconnaît l'apport de Kierkegaard, celui qui a sans cesse insisté sur la proximité de Dieu dans le prochain. Un parallèle au sujet de la conception de la vérité pourra s'avérer instructif puisque Levinas paraît accepter la thèse kierkegaardienne de la subjectivité comme séparée, comme non vérité, qu'il critiquait en 1961. Sept ans plus tard, la question du dieu-homme ne semble pas pouvoir se poser autrement. Dans ce texte, l'idée de «transcendance dans la non certitude » est assumée par Levinas. Cela est d'autant plus pertinent si on se rappelle que chez Kierkegaard, qui est épris des Écritures (Levinas ne le sera pas moins), la vérité persécutée et humiliée est une autre manière de penser la vérité en dehors de la 
philosophie. La vérité n'est plus une affaire d'objectivité, mais une preuve de la transcendance, de la séparation. Levinas relève un point des plus importants pour nous : chez Kierkegaard, la persécution apparaît comme une «modalité du vrai » et nous dirons que c'est peut-être sa plus haute expression. Cette modalité implique une conception précise de la transcendance.

Si Kierkegaard défendait une subjectivité comme non vérité, insistant sur le refus d'un absolu immanent à soi-même, il soulignait que l'homme est par nature non-liberté. Si l'homme, dans le cadre de la «double réflexion » présentée plus haut, peut rencontrer l'absolu comme absolument autre que lui-même et transcendant à lui, c'est parce que l'absolu vient à lui en empruntant sa condition d'homme, se révélant par sa parole, lui indiquant par là sa différence incommensurable. Ainsi compris, le paradoxe absolu est bien le moteur de la pensée de la «double réflexion » de Kierkegaard. Levinas, qui ne semblait pas refuser le scandale au sujet du paradoxe, ne parvient pas à reconnaître le travail de la double réflexion kierkegaardienne qui passe de l'intériorité à l'extériorité et qui, dans un second mouvement, revient de l'extériorité vers l'intériorité afin d'affirmer et de valider une action du sujet face à Dieu. L'individu kierkegaardien n'accomplit pas simplement un mouvement d'ascension vers un être transcendant, ni davantage ce mouvement en approfondissant en lui-même (dans l'intériorité pure ou le secret que dénonce Levinas) une vérité le dépassant, c'est dire qu'il n'extrait pas de lui-même la vérité, mais qu'il la rencontre en la recevant d'un être transcendant tout autre que lui.

\section{L'éthique de la proximité aux limites du religieux}

Malgré des méthodes philosophiques et des croyances religieuses différentes, Levinas et Kierkegaard s'entendent sur plusieurs points. Nous en retiendrons ici au moins deux, lesquels 
pourront servir à penser une éthique de la proximité à partir de ces deux philosophies. (1) La proximité que l'on cherche à gagner avec l'Autre, le prochain, se trouve dans une tension, un rapport, un écart, une distance constituante de la subjectivité elle-même dans l'existence. Cette proximité chez les deux auteurs ne se pense pas en dehors d'un rapport à une transcendance. (2) Le prochain, qui est absolument Autre que moi, se trouve en même temps en moi. S'il est bien sûr à l'extérieur de moi, je pourrai le rejoindre en vertu de mon intériorité et de mon visage. Ce prochain n'entre pas dans le discours de la conscience en quête de thématisation et de détermination; il est bien plutôt dans le visage insaisissable de l'Autre.

\section{(a) L'éthique de la proximité confrontée à l'indicible}

L'éthique, on l'a vu, appelle un autre langage, un langage sensible aux limites du Dire. Le discours du savoir, qui vise à nommer l'autre, nous en détourne. Et c'est ici que l'objection au système par Kierkegaard rejoint le geste philosophique fondamental de Levinas, ce qui aboutit dans une critique de l'ontologie qui transforme le Dire en Dit. Dans ce contexte où la réflexion porte sur l'Autre comme différence, comme distance et corps, le visage (son épiphanie ${ }^{20}$ ) apparaît d'une certaine manière à Levinas comme l'expression de l'intériorité et l'intériorité de l'expression. On pourra dire aussi qu'il s'agit d'une reprise de la dialectique de la distance éthique kierkegaardienne : le prochain est en moi et m'oblige à une compréhension juste de la distance qui me sépare de lui. Trop près, je ne le vois pas, et trop loin, il ne semble plus être mon prochain. Par le langage, je peux m'approcher ou m'éloigner d'autrui. Kierkegaard, qui avait utilisé l'idée du visage avant Levinas, ne serait pas déçu de son interprète, car il rend justice à la dialectique de la distance qui affirme que dire «me voici » peut être un éloignement de l'éthique. 
On retiendra finalement que l'éthique, au sortir d'une confrontation entre deux des plus grands penseurs de la singularité, ne pourra plus faire l'économie du concept de distance, encore moins de celui de proximité, puisque la vérité de la subjectivité, qu'elle soit souffrance dans la distance avec Dieu ou l'expression de l'interdiction dans le visage de l'Autre, repose sur la capacité de se poser dans l'existence. Dans l'existence toujours singulière, l'individu n'accomplit pas un mouvement direct vers les autres, encore moins un mouvement d'ascension total vers l'être transcendant. Au contraire, lié aux autres, il approfondit en lui-même une vérité transhistorique le dépassant, une idée comme l'infini de Levinas. Ainsi est-il à même de rencontrer cette vérité en la recevant d'un être Tout Autre que lui, pour parler cette fois avec Kierkegaard. Cherchant à dépasser Kierkegaard, devons-nous conclure, Levinas s'impose comme l'un de ses meilleurs commentateurs.

\section{Notes}

1 Nous remercions les membres du comité de lecture de PhaenEx pour leurs excellentes suggestions.

2 Dans le cadre de cet article, nous ferons référence, outre les textes cités, à des passages de l'œuvre de Levinas où il est fait explicitement allusion à Kierkegaard, c'est-à-dire à Levinas, «Kierkegaard - Existence et éthique » et « À propos de Kierkegaard vivant», dans Noms propres 99-109. Les autres rapprochements entre les auteurs reposeront strictement sur la communauté des thèmes et des manières communes de les traiter. Notons en passant que dans «Violence et métaphysique » (L'écriture et la différence, 1979), Derrida avait déjà attiré l'attention sur la polémique entre Kierkegaard et Levinas au sujet de la responsabilité et du fameux «me voici! ».

3 Il importe de souligner que Levinas ignore l'œuvre de Kierkegaard. J. Colette précise ce fait important en ces termes : "Sauf dans l'article de 1977, Levinas ne se réfère pas aux textes mêmes de Kierkegaard, il reprend les thèmes et les formulations de Wahl » («Levinas et Kierkegaard»6, note). Colette insiste non sans raison: «Par cette réfutation simultanée de Hegel, de Heidegger et d'un Kierkegaard réduit au "subjectivisme pur du moi" (TI, XIV), 
Levinas ne se réfère qu'au Kierkegaard lu par J. Wahl, au chrétien réduit à la figure de la "conscience malheureuse", à "l'amant malheureux de la religion" » (11).

D. Brezis soulève aussi notre objection aux accusations de Levinas. Il écrit : «Or il est remarquable qu'à travers ces deux points il accuse justement Kierkegaard de ce qu'il entend récuser. [...] Il serait complice de la violence que le Même exerce sur l'Autre ( «L'intériorité en question »17). Travaillant le regard croisé entre les auteurs à partir de sa méthode catégoricobiographique, Brezis, pour de nombreuses raisons qu'il expose en page 18, trouve peu convaincantes les critiques de Levinas, que ce soit celles présentes dans Totalité et infini ou celles de Noms propres.

${ }^{5}$ Nous appellerons en renfort l'article de P. Søltoft dans lequel l'auteure dénonce la réduction opérée par Levinas («Love and Continuity» 215). Søltoft rappelle que la critique de Levinas est une erreur ou une méprise philosophique, car elle s'interprète uniquement dans la direction d'un individu qui, laissant de côté la construction progressive du moi par l'amour et la continuité, est incapable de rejoindre autrui. La vérité est ailleurs. Levinas, selon Søltoft, a oublié le caractère intersubjectif du concept de continuité éthique fondée dans une doctrine de l'amour. L'idée de « tension sur soi », qui n'est pas mauvaise en soi, cache une distance fondamentale, non pas uniquement celle entre le moi et les autres, mais aussi et davantage celle entre l'homme et luimême, celle qui fonde l'éthique (224-227).

${ }^{6}$ Il écrit : "In this way, the thematisation of the I and the Other in their relation must always miss the mark: it must always be undone, unsaid, for any such thematisation must always miss what lies behind thematisation itself» («Philosophy Always Comes too Late »161). Or sur la thèse de M. Weston et le rôle de la temporalité dans la thématisation, il écrit : "For in being spoken of, the attempt is made to make it present, to disclose the matter in what is said. But the primal relation is what lies beyond and behind all thematisation in a time which is other than the time within which what lies in the future can appear in the present and be recollected as having been in the past : it is always having occurred in a past more ancient than any present, a past which was never present. [...] But the thematisation, the formulation of the saying in the said, here fails irredeemably » (165).

${ }^{7}$ Le titre de l'article de M. Weston vient directement d'un passage d'Autrement qu'être. Levinas écrit en effet dans la section consacrée au problème de la proximité : "Le connaître est toujours convertible en création et en anéantissement, l'objet se prêtant au concept, en résultat. Par la suppression du singulier, par la généralisation, le connaître est idéalisme. Dans l'approche je suis d'emblée le serviteur de prochain, déjà en retard et coupable de retard » (Autrement qu'être 139). Levinas, pour prendre un autre thème d'Autrement qu'être, dit souvent que l'obsession et la persécution qualifient l'in-condition d'une conscience de soi en retard par rapport à autrui (174176).

8 Sur le thème commun du témoignage, lire Janiaud, «"Me voici!" » 100-105, et Brezis, «L'intériorité en question » 24. 
${ }^{9}$ Sur ce point décisif, voir Crainte et tremblement, mais aussi la Reprise de Kierkegaard (CEuvres complètes V). Pour le commentaire, voir notre article «The Exception as Reinforcement of the Ethical Norm ». Pour une lecture originale de l'exception, consulter l'excellent article de S. de Keyser, « Kierkegaard et l'exception ».

${ }^{10}$ Un article récent s'est employé à montrer que cette association rapide entre le repli sur soi, l'égoïsme, la violence et l'irresponsabilité n'était pas fondée (Janiaud, «"Me voici!" » 90-92).

${ }^{11}$ Au sujet des limites des attaques de Levinas et de la question de la distance éthique, voir nos Expressions éthiques de l'intériorité, sections III et IV.

${ }^{12}$ Sur ce thème précieux pour Kierkegaard, voir l'entrée XIII A 128 intitulée « Chair et sang le langage », dans les Papirer. Ici, nous ne prétendons pas que Levinas ait étudié les Papirer, mais que les termes sont exactement les mêmes. Pour expliquer ces liens étroits ou cette coïncidence, on invoquera que la réception kierkegaardienne, notamment chez Rosenzweig, a peut-être donné des indications assez précises à Levinas. Pour une interprétation de cette entrée, lire nos Expressions éthiques de l'intériorité 228.

${ }^{13}$ Pour le bien de la démonstration, nous rappellerons un passage de la dialectique de la compréhension à distance des Kjerlighedens Gjerninger. Kierkegaard écrivait : «À distance, tous connaissent le prochain [kjender Enhver Naesten]; mais Dieu seul sait combien le connaissent en réalité, de près. Et pourtant, à distance [paa Afstand], le prochain, lui qui est par définition le proche, le premier venu, absolument tout homme est une illusion » (Euvres complètes XIV 70 [IX 92]).

${ }^{14}$ Levinas s'est toujours beaucoup intéressé à la question juive. Pour mieux comprendre sa position, lire Difficile liberté.

${ }^{15}$ Afin d'obtenir une autre interprétation de ce passage, voir Brézis, «L’intériorité en question ».

${ }^{16}$ Cette lecture de Nizet, on le regrette, oppose radicalement intériorité et extériorité.

${ }^{17}$ Concernant la critique adornienne de Kierkegaard, on lira la thèse d'habilitation de 1933 traduite en français: Adorno, Kierkegaard. Pour un commentaire de la réception, voir Desroches, «Esthétique, musique et langage ».

18 Levinas, qui ne parvient pas à reconnaître le travail de la double réflexion, accomplit cependant un renversement en sa pensée, bien que différent de celui de Kierkegaard. En effet, on reconnaîtra par exemple que dans la substitution, il y a bien un renversement, un passage du par l'autre au pour l'autre. Nous irons même plus loin : il y a un retournement dans l'être, c'est-àdire dans l'autrement qu'être soi-même. Dans l'œuvre en général, on assiste aussi à un retournement lorsque, de Totalité et infini à Autrement qu'être, l'Autre dans le Même semble mettre l'accent sur l'extériorité radicale. 
19 Pour donner une idée de La dialectique de la communication de 1847 et du reproche que Kierkegaard adresse à ses contemporains, citons un passage clair : «La confusion fondamentale du monde moderne consiste non seulement à avoir oublié la réalité de ce que l'on nomme la communication de pouvoir, mais à avoir absurdement transformé la communication de pouvoir et de devoir-pouvoir en communication de savoir. Le facteur existentiel a été éliminé » (Euvres complètes XIV, 389).

${ }^{20}$ L'épiphanie du visage vient d'une certaine manière briser la réclusion du moi. Car le visage est d'abord une expression du mode éthique. Sur l'épiphanie du visage, relire Totalité et infini, notamment la section III consacrée au visage et à l'extériorité. Pour un commentaire introductif à la question de l'épiphanie du visage, voir Plourde, Avoir-l'autre-dans-sa-peau 43-59.

\section{Ouvrages cités}

Adorno, T. W. Kierkegaard. Construction de l'esthétique. Trad. E. Escoubas. Paris: Payot, 1995.

Brezis, D. «L'intériorité en question. Regards croisés sur Kierkegaard et Levinas ». Rue Descartes, $\mathrm{n}^{\circ}$ 43, 2004: 16-28.

Calin, R. Levinas ou l'exception du soi. Paris: Epiméthée, 2005.

Colette, J. «Levinas et Kierkegaard. Emphase et paradoxe». Revue philosophique de Louvain, février-mai 2002: 4-31.

Chevalier, P. «Abraham et le commandement de l'amour chez Abraham». Archives de philosophie, vol. 67, 2004: 321-334.

Clair, A. Kierkegaard. Existence et éthique. Paris: P.U.F., 1997.

Danta, C. «The Poetics of Distance : Kierkegaard's Abraham ». Literature \& Theology, 2007: 118.

Desroches, D. «The Exception as Reinforcement of the Ethical Norm : the Figures of Abraham and Job in Kierkegaard's Ethical Thought ». In C. Daigle (dir.). Existentialist Thinkers and Ethics. Montréal/Kingston: Mc Gill/Queen's University Press, 2006: 24-36.

—. «Esthétique, musique et langage. Retour sur la réception critique de Kierkegaard par T. W. Adorno ». Horizons philosophiques, vol. 16, $\mathrm{n}^{\circ}$ 2, 2006: 21-38.

—. Expressions éthiques de l'intériorité. Éthique et distance chez Kierkegaard. Québec: P.U.L., 2008 (coll. Inter-sophia). 
Janiaud, J. «"Me voici!" Kierkegaard et Levinas : les tensions de la responsabilité ». Archives de philosophie, vol. 60, $\mathrm{n}^{\circ} 1,1997: 87-108$.

Jegstrup, E. «Kierkegaard on Abraham's Tragedy: the Loss of Community ». PhaenEx, vol. 1, $\mathrm{n}^{\circ}$ 2, 2006: 21-46.

Kemp, P. «Éthique et langage : de Levinas à Kierkegaard». Les Cahiers de philosophie, vol. 8/9, 1989: 187-210.

—. L’irremplaçable. Paris: Cerf, 1993.

Keyser, S. de. «Kierkegaard et l'exception». Revue philosophique de Louvain, vol. 104, n 3, 2006: 467-497.

Kierkegaard, S. A. Euvres complètes. Trad. P.-H. et E.-M. Tisseau. Paris: éd. de l'Orante. 20 vol. 1966-1986.

—. Papirer. P. A. Heiberg, V. Kuhr et E. Torsting (dir.). Copenhague: Gyldendal._20 vol. 19091948 / 2 Udg. N. Thulstrup. Copenhague. 1968-1978.

-. Samlede Varker. A. B. Drachmanm, J. L. Heiberg et H. O. Lange (dir.). Copenhague: Gyldendal. 1920-1936.

Levinas, E. « À propos de Kierkegaard vivant» et «Kierkegaard. Existence et éthique ». In Levinas. Noms propres. Paris: Fata Morgana, 1987.

—. Autrement qu'être ou au-delà de l'essence. La Haye: Nijhoff, 1974.

—. Difficile liberté. Paris: Albin Michel, 1963.

—. Entre nous. Paris: Grasset, 1991.

—. Éthique et infini. Paris: Fayard, 1982.

—. «Langage et proximité » (1967). In Levinas. En découvrant l'existence avec Husserl et Heidegger. Paris: Vrin, 1994.

—. Totalité et infini. Essai sur l'extériorité, La Haye, Nijhoff, 1961.

Nizet, J. «La temporalité chez Soeren Kierkegaard». Revue philosophique de Louvain, vol. $71, \mathrm{n}^{\circ} 2,1973$.

Plourde, S. Avoir-l'autre-dans-sa-peau. Lecture d'Emmanuel Levinas. Québec: P.U.L., 2003. 
Ricoeur, P. Autrement. Lecture d'Autrement qu'être d'Emmanuel Levinas. Paris: P.U.F., 1997.

Soltøft, P. «Love and Continuity : the Significance of Intersubjectivity in the Second Part of Either/Or ». Kierkegaard Studies. Cappelhørn \& Deuser (dir.). Berlin: de Gruyter, 1997: 210-227.

Wahl, Jean. Études kierkegaardiennes. Paris: Aubier, 1938.

Westphal, M. «The Transparent Shadow. Kierkegaard and Levinas in Dialogue ». In M.J. Matuštík et M. Westphal (dir.). Kierkegaard in Post/Modernity. Indianapolis: Indiana University Press, 1995.

Weston, M. «Philosophy Always Comes too Late. Levinas and Kierkegaard ». Kierkegaard and Modern Continental Philosophy. London: Routledge, 1994: 156-177. 